\title{
I MERCANTI RAGUSEI E LE CROCIATE DEL TARDO MEDIOEVO FINANZIAMENTI PER LA GUERRA E LASCITI PRO ANIMA: ... AD PASAGIUM TURCHORUM SEU SARACENORUM, ET ALLIUD SUPER PATARENOS BOSNIE
}

\author{
RAGUSAN MERCHANTS AND THE CRUSADES IN THE LATE MIDDLE AGES. \\ FINANCIAL SUPPORT FOR THE WAR AND BEQUESTS PRO ANIMA \\ ... AD PASAGIUM TURCHORUM SEU SARACENORUM, \\ ET ALLIUD SUPER PATARENOS BOSNIE
}

\author{
DANIELA ROMANO \\ Università S. Orsola Benincasa \\ Napoli (Italia)
}

\begin{abstract}
Riassunto: I testamenti ragusei del XIII e XIV secolo rivelano aspetti particolari e per nulla indagati sul pellegrinaggio armato per procura in area balcanica. La lotta agli infedeles e agli eretici messa in atto dalla Chiesa di Roma trova sostegno economico e piena adesione nei cittadini di Dubrovnik, città-stato baluardo della cristianità più a levante, anche grazie alla campagna pubblicistica attuata da francescani e domenicani. Nobili, mercanti, chierici e preti, artigiani, uomini e donne partecipano alla giusta guerra, prevedendo lasciti per uomini che combatteranno per la salvezza delle anime dei testatori.
\end{abstract}

Parole chiave: Dubrovnik; Medioevo; Crociate; Eretici; Testamenti.

\begin{abstract}
Ragusan wills of 13th and 14th centuries reveal peculiar and unsuspected aspects about armed pilgrimages by proxy in the Balkans. The struggle against infedeles and heretics propagandized by the Roman Church finds financial support and full agreement in Dubrovnik citizens, also thanks to the Franciscan and Dominican preaching. Nobles, merchants, clerics and priests, craftsmen, men and women, they are all ready to take part in the Just war. Anyway they would fight only on the condition of leaving a sum of money to those who would fight for their souls' salvation.
\end{abstract}

Keywords: Dubrovnik; Middle Ages; Crusades; Heretics; Wills.

Dico cur robavi et fui malus christianus et violentus... confessa nel suo testamento olografo all'alba del 3 febbraio 1348 il nobile e ricco cittadino di Dubrovnik Bene de Gondola, figlio di Marino'. L'ingente patrimonio di questo esponente di una delle famiglie più prestigiose ed influenti della città - perchè mihi reprendet consciencia - viene diviso e destinato quasi per intero alla costruzione di cappelle e romitori nel territorio di Ragusa, a messe che frati predicatori e frati minori dovranno cantare pro anima nei monasteri e nelle chiese della città, all'acquisto di libri e arredi sacri, e al compimento di

${ }^{1}$ ASD (Archivio Storico di Dubrovnik), Testamenta de notaria, serie X.1, vol. 3, ff. 77 r. v. 
pellegrinaggi vicari nei maggiori centri della cristianità in occidente (Roma, Assisi, S. Giacomo di Compostella, S. Nicola di Bari e Montesantangelo).

Gli elementi fin qui elencati si inseriscono in un quadro ben noto e indicativo della mentalità, ma soprattutto della spiritualità medievale. Ciò che invece desta il nostro interesse è la particolare clausola testamentaria che il Gondola inserisce quasi alla fine del documento e che prevede un lascito di 140 perperi (circa 70 ducati veneziani di oro) a due uomini causa eundi ad pasagium turchorum seu saracenorum, et alliud super patarenos Bosnie et quousque fuerit passagium: una investitura di fatto a partire per la guerra santa.

Questo testamento fa parte di una serie documentaria, denominata Testamenta de notaria, conservata presso l'Archivio di Dubrovnik e costituita da 94 volumi rilegati e distribuiti su di un arco cronologico che va dal XIII al XIX secolo ${ }^{2}$. Di questi solo 26 sono i volumi relativi al Medioevo e i primi sette riportano atti databili dal 1282 al 1391: si tratta di copie redatte per la Cancelleria, testamenti rogati nella maggior parte dei casi da notai e giudici alla presenza del Rettore della città e di testimoni ${ }^{3}$. Quasi la metà dei testamenti, circa 150 atti, si concentra tra il 1348 e il 1363, gli anni delle epidemie di peste. Inoltre su un totale di 394 atti, ben 163 registrano le ultime volontà di donne appartenenti all'aristocrazia mercantile cittadina, alla borghesia e al popolo, tutte indistintamente impegnate a trasmettere i patrimoni e che sembrano avere un ruolo economico ben definito nella gestione di beni e legami familiari, di investimenti e conservazione delle ricchezze.

Emergono da questa particolare fonte documentaria le motivazioni, le mete, i compiti e gli oneri economici dei pellegrinaggi ma, soprattutto, emerge il quadro di una società cittadina ricca ed eterogenea costituita, nel XIV secolo, approssimativamente da 4000 persone $^{4}$, i cui rapporti politicodiplomatici ed economici e commerciali con la costa adriatica dell'Italia, con gli stati italiani, con la penisola iberica e gli altri potentati dell'Europa

${ }^{2}$ Il testamento è una delle numerose fonti utilizzate nell'ambito di una ricerca più vasta attualmente in corso, relativa al pellegrinaggio raguseo nel XIII e XIV secolo condotta nell'ambito del Dottorato di ricerca in "Storia del centri, delle vie e della cultura dei pellegrinaggi nel Medioevo euromediterraneo" dell'Università di Lecce.

${ }^{3}$ Sono limitati e abbastanza datati gli studi e le informazioni relative all'organizzazione del notariato raguseo. Era indubbiamente un ufficio dello stato e i registri facevano parte dell'archivio della cancelleria della repubblica a partire dal 1278. Dopo il 1284 la cancelleria statale fu divisa in Cancellẹiạ e Notariato e quest'ultimo tornọ nellẹ mani dẹi religiosi locali almeno fino al 1324 , anno in cui si cominciarono ad assumere colti notai stranieri. A tal riguardo cfr. Ignacji VoJE, Il credito nella Ragusa medievale, pp. 45-60, in Ragusa e il Mediterraneo, a c. di Antonio DI VITTORIO, Bari, 1990; Joan DUSA, The medieval Dalmatian episcopal cities: development and transformation, New York, 1991; Ante MARINOVIć, Les origines et les premiers actes des chancelleries urbane en Dalmatie, en particulier a Dubrovnik et Kotor (XIIIe-XIVe s.), in Gabrie SILAGI, Landesherrliche Kanzleien im Spätmittelalter, Münchener Beiträge zur Mediävistik und Renaissance-Forschung, München, 1984, p. 587 ss.

${ }^{4}$ Cfr. Barisa KREKIC, Contributions of foreigners to Dubrovnik's economic growth in the late middle ages, in Dubrovnik, Italy and the Balkans in the Late Middle Ages, XIX, pp. 375-394, Londra, 1980 
occidentale e orientale, sono ben documentati e studiati ${ }^{5}$. Restano invece largamente insufficienti, sotto il profilo quantitativo, gli studi sul pellegrinaggio in area levantina, soprattutto croata, dove si segnalano, infatti, lavori riferibili solo a piccole realtà, quali Traù, Zara e Spalato ${ }^{6}$.

La storiografia croata ha mostrato interesse per la fonte testamentaria soprattutto a partire dall'ultimo trentennio, con l'obiettivo di indagare sulla devozione popolare, sulle usanze funerarie e sul rapporto con la morte e, in particolare, per evidenziare aspetti della vita quotidiana e della cultura materiale medievale, non solo quella specifica delle singole realtà locali cittadine, ma confrontandole a quelle regionali ed europee ${ }^{\gamma}$.

${ }^{5}$ Vasta è la bibliografia su Dubrovnik nel medioevo. Si rimanda pertanto ai lavori del Krekic e al ricco repertorio bibliografico da lui esaminato nell'Introduzione al saggio: Barisa KREKIC, Dubrovnik: a Mediterranean Urban Society, 1300-1600, Londra 1997, in particolare le pp. IX'XVIII; cfr. anche il testo più divulgativo di Robin HARRIS, Dubrovnik. A History, Londra, 2003. Per i rapporti con il Papato, cfr. il lavoro di Kenneth SETTON, The Papacy and the Levant (1204 1571), vol. I, The Thirteenth and Fourteenth centuries, Philadelphia 1976; per i rapporti con l'entroterra, cfr. Ivan DUJCEV, Rapporti fra Venezia e la Bulgaria nel Medioevo, in Venezia e il Levante fino al secolo XV, a c. di Agostino PERTUSI, Firenze, 1973, pp. 237-259; e ancora Barisa KREKIC, Le relazioni tra Venezia, Ragusa e le popolazioni serbo-croate, in Venezia e il Levante, cit., pp. 389-401.

${ }^{6} \mathrm{Cfr}$. Zoran LADIĆ, Prilog proučavanju hodočašcenja iz Zadra u drugoj polovici 14. stoljeća (Contributo allo studio del pellegrinaggio di Zara nella seconda metà del XIV secolo), «Croatica Christiana Periodica», 32/XVII (1993), Zagreb, in cui lo studioso elenca i contributi degli ultimi anni e cita i lavori di Slobodan NovAK, Hodočasnik Santo Brasca na Hvaru 1480. godine (Il pellegrinaggio di Santo Brasca nel 1480), in "Hvarski zbornik», sv. VI, Hvar 1978; di Josip KOLANOVIC, Prilog povijesti šbenskih hodočaśća u kasnom srednjem vijeku, in "Croatica Christiana Periodica», VI (1982), Zagreb, pp. 13-36; e infine quello di J. RICHARD, Križari putnici u srednjovjekovnoj. Slavoniji (1 crociati e i viaggiatori nella Slavonia del medioevo) in "Croatica Cristiana Periodica», X (1986), fasc. 18, Zagreb, pp. 27-39. Per la Slovenia ricorda $\mathrm{i}$ lavori di Ignacij VoJE, Romanje Ulrika II Celjskog v Kompostelo $k$ sv. Jacobu (Il pellegrinaggio di Ulrik da Cilli a Santiago de Compostela), in «Zgodovinski casopis», 38 (1984), Ljubljana, pp 225-230; Ferdo GESTRIN, Piranska potovanja v 15. stoletju (I pellegrinaggi a Pirano nel XV secolo), in «Kronika. Casopis za slovensko krajevno zgodovino», XXIII (1975), Ljubljana, pp. 74-80. Questi pochi lavori croati e sloveni "permettono comunque di effettuare determinati confronti nello studio del pellegrinaggio della costa orientale adriatica". Da segnalare ancora sono i lavori di N. BUDAK, Hrvatska hodoćaśća u Aachen (I pellegrinaggi croati ad Aquisgrana), in «Radovi», Zavod za Hrvatsku Povijest (Istituto di Storia Croata), vol. 24, Zagreb 1991, pp. 1521; S. KRASIĆ, Opis hrvatske jadranske obale u putopisima svicarskog dominikanca Feliksa Fabrija (Schmida) iz 1480. I 1483/84 godine (Descrizione della costa croata nel racconto di viaggio del frate svizzero domenicano Felix Fabri fatto nel 1480 e nel 1483/84), in "Anali Dubrovnik», 39 (2001), Dubrovnik, pp. 133-216; Zoran LADIĆ, Odjek pada tvrdave Accon 1291. g. $u$ hrvatskoj (L'eco della caduta di Acri nel 1291 in Croazia. La crociata come forma di pellegrinaggio), in ZPDZ, 16 (1998), Zagreb, pp. 43-55; Zoran LADIĆ, Legati kasnosrednjovjekovnih dalmatinskih oporuçitelja kaomizvor za proučavanje nekih oblika svakodnevnog zvota $i$ materijalne kulture (I lasciti dei testatori dalmati del tardo Medioevo come fonte per lo studio di alcune forme di vita quotidiana e di cultura materiale), in «Odsjek za povijesne znanosti», HAZU 21 (2003), Zagreb; Nikola Mate RoŠCIC̣, Hrvatska hodocascenja u Loreto (I pellegrinaggi croati a Loreto), in "Croatica Christiana. Periodica», VII (1983), fasc. 11, Zagreb, pp. 88-96; Maria KARBIC, Zoran LADIC, Oporuke stanovnika grada Trogira u arhivu HAZU (I testamenti di Traù nell'archivio dell'Accademia Croata di Scienze ed Arti), in «Radovi. Zavoda za povijesne znanosti HAZU u Zadru», 43 (2001), pp. 161-254.

${ }^{7}$ Per un panorama approfondito sullo stato degli studi, cfr. Zoran LADIĆ, Oporućni legati pro anima i ad pias causas u europskoj historiografijl. Usporedba s oporukama datmatinskih komuna (I legati testamentari pro anima e ad pias causas nella storiografia europea. Comparazione con 1 testamenti dei comuni dalmati), in ZDPZ HAZU, 17 (1999), Zagreb, pp. 17-28. Lo stesso autore analizza i lasciti dei testatori dalmati in relazione all'abbigliamento e ai tessuti, al cibo e a quant'altro attiene alla cultura materiale e sottolinea come, a partire dalla seconda metà del XIII secolo, cambia sia la tipologia dei legati sia la scelta dei destinatari. Nei comuni dalmati, infatti, $\mathrm{i}$ beni in denaro o terre lasciano il posto a quel poco che i testatori possedevano nella propria casa, dai mobili ai capi di abbigliamento. Cfr. Zoran LADIĆ, Legati kasnosrednjovjekovnih, cit., pp. 1-28. 
Il testamento è l'ultimo atto volontario della vita terrena, è il momento in cui se per un verso status, dignità sociale, ruoli, acquistano una fortissima colorazione religiosa e "democratica", per l'altro la volontà di perpetuare la memoria di sé, il desiderio o la necessità di sistemare le faccende dell' "aldiquà", lo rendono elemento fondamentale per evidenziare una rete sociale, spirituale, e principalmente economica di primaria importanza. Nobili e popolani, magnati e meno abbienti, tutti organizzano i loro beni per la famiglia e pro remedio animae e nessuna fonte è più originale di quella testamentaria per indagare sulla metafora del pellegrinaggio.

E' particolarmente indicativo che negli atti esaminati tutte le tracce relative al pellegrinaggio, incluso quello "armato", rimandano alla tipologia della procura: i testatori, cioè, non partono per i luoghi santi o per la crociata ma, come il nostro mercante Bene de Gondola, piuttosto destinano una somma perché qualcuno - preferibilmente preti o almeno di boni e honesti homines, degni insomma di fede- si rechi ad espiare i loro peccati nei luoghi designati. Dai testamenti ragusei emerge quella «mentalità oggettivistica -noi aggiungeremmo anche utilitaristica-, tipica del Medio Evo, per cui ciò che contava era l'opera da compiere, poco importava della persona, di chi cioè la doveva compiere ${ }^{8}$. Pertanto la carica di spiritualità è direttamente proporzionale alle somme di denaro lasciate, al numero di viaggi richiesti, ma anche al valore e all'importanza che occupano negli atti le clausole.

Gran parte delle disposizioni patrimoniali di Dubrovnik in questo periodo descrivono un impegno per lo più mercantile i cui interessi e le cui attività coprono svariati settori- dal commercio di cabotaggio all'argento, dai panni alla cera, ma che non trascurano il tradizionale investimento terriero (nella maggior parte dei casi vigneti), fino ad arrivare al prestito su pegno: di sicuro si può affermare che ognuno di loro sente con orgoglio l'appartenenza alla piccola repubblica, siano essi cittadini o solo habitatores. E' indubbiamente una città «di mercanti... per i mercanti», per usare la calzante definizione del Lopez ${ }^{9}$, una città che riesce sempre a garantire la sicurezza personale e il diritto al libero commercio ai suoi cittadini dovunque si trovino ${ }^{10}$.

Le fonti testamentarie prese in considerazione forniscono - in questo particolare contesto storico e geografico- un elemento nuovo, quello appunto dei lasciti in denaro o in armi destinati al pellegrinaggio armato "per procura" che, per usare una acuta definizione del Cowdrey, permise alla Chiesa di essere "in contatto diretto con le armi senza passare attraverso la mediazione dei re" $" 11$ per circa cinque secoli (XI-XVI).

\footnotetext{
${ }^{8}$ Mario SENSI, Pellegrinaggi a Montesantangelo al Gargano nei notarili della valle spoletana sul calare del Medioevo, "Campania Sacra. Studi e Documenti», VIII-IX (1980), pp. 81-120, p. 85 .

${ }^{9}$ Roberto LóPEZ, Su e giù per la storia di Genova, Genova, 1975, p. 36.

${ }^{10}$ Radovan SAMARDŽIĆ, Ragusa come sistema di funzioni, pp. 3-11, in Antonio DI VITTORIO, Ragusa e il Mediterraneo, Bari, 1990.

${ }^{11}$ Herbert Edward John CowDREY, The Genesis of the Crusades, in The Holy War, a c. di Thomas MURPHY, Columbus, 1976, p. 16.
} 
Questo particolare aspetto riconduce al tema della conflittualità medievale e più in generale ai criteri della "giusta guerra" che, definiti con precisione circa un secolo prima dalla Scolastica e in particolare da Tommaso d'Aquino nella sua Summa Theologiae ${ }^{12}$, furono messi in pratica a partire dalla prima crociata indetta da Urbano II. Dai primi anni dell'XI secolo, quando Pier Damiani affermava che in nessuna circostanza era consentito prendere le armi per la difesa della fede della Chiesa universale e che, ancor meno, gli uomini potevano dar battaglia per beni terrestri e transitori, si arrivò al 1140, anno in cui il giurista Graziano asseriva invece non essere peccato fare la guerra giacché «una guerra è giusta se è condotta con intenzione positiva, sotto la direzione di un'autorità legittima e con scopo difensivo o con lo scopo di recuperare un bene ingiustamente preso». Tra XI e XII secolo nasce dunque una nuova sensibilità religiosa che pone sullo stesso piano penitenziale sia l'abbracciare una vita eremitica, sia il partire per un pellegrinaggio armato e combattere gli infedeli. Come sottolinea la Muzzarelli «molta strada separava l'antico ideale cristiano del martirio per fede, dall'idea della guerra santa nata dopo la riforma della Chiesa dell'XI secolo; da allora alla lotta contro il corpo per vincere le tentazioni e trionfare sul peccato si affiancò una nuova forma di penitenza: la prassi della guerra a difesa della fede» ${ }^{13}$.

E' evidente dunque che i testatori ragusei -mercanti, donne, ecclesiastici, artigiani, semplici popolani- aderiscono pienamente e fanno proprio questo concetto di penitenza: pertanto il bellum iustissimum è azione virtuosa e meritevole di uno speciale favore divino, capace di rimettere le pene. Già nel 1284 il nobile e ricco mercante Elia de Rasti ${ }^{14}$ devolve nel suo testamento 20 perperi e la sua panceria quando fuerit pasaçium generale ultra mare usque ad quatuor annos proxime venturos. Ma il valore espiatorio del lascito viene suggerito dalla clausola che prevede di destinare quella stessa somma alla consacrazione della chiesa di S. Elia de Tumba e alla celebrazione di messe, nel caso non fosse stata indetta alcuna crociata nell'arco dei quattro anni. Elia de Rasti si dimostra non solo in questo caso pragmatico: il resto del suo patrimonio deve essere dato ad navigandum ut videbitur piropi meis, ad lucrandum cum ipsa in modo che le due figlie possano sposarsi. Alla sua morte, rende liberi e franchi i suoi due schiavi, Bosiça e Radoslavus.

Gli altri testamenti ragusei del XIII secolo, relativi al pellegrinaggio armato per procura, si concentrano negli anni 1295-1299. Occorre peraltro ricordare una serie di eventi che avevano favorito questa prassi: nel 1240, papa Gregorio IX aveva permesso di sostituire col denaro la promessa di partire per la crociata, e questo non solo nel caso dell' impedimento fisico di chi li aveva fatti; tra la fine del XIII secolo e l'inizio di quello successivo, i

\footnotetext{
${ }^{12} \mathrm{~A}$ tal proposito cfr. Frederick H. RusSELL, The Just War in the Middle Ages, Cambridge, 1975; Philippe CONTAMine, La guerra nel Medioevo, Bologna, 1986.

${ }^{13}$ Maria Giuseppina MuZzarelli, Penitenze nel Medioevo. Uomini e modelli a confronto, Bologna, 1994, p. 114s.

${ }^{14} \mathrm{ASD}$, Testamenta, vol. 1, ff. 25v.-26r.
} 
frati incaricati nel continente di predicare la guerra, ebbero la facoltà di commutare in 200 tornesi la promessa di combattere l'infedele. In siffatto cambio di prospettiva appare del tutto condivisibile quanto detto in proposito dal Sumption: «se le indulgenze avevano insegnato agli uomini a considerare il merito una merce, non era del tutto innaturale che essi lo ritenessero un bene trasferibile» ${ }^{15}$ !

E non meraviglia dunque che questa certezza della trasferibilità dell'indulgenza - anche quando l'effusio sanguinis è commissionato ad altriinveste anche i chierici, la cui militarizzazione era in atto fin dal IX secolo. A prova di ciò, nel 1295 il chierico Rosino Balislava ${ }^{16}$ di Dubrovnik elenca nel testamento le sue armi destinandole alla Crociata: ...panceria, insegna, lamera et spata mea bona, capilina et scutum dentur ultra mare ... si pasagium fuerit mitatur homo unus pro uno anno cum illis armis ... (in conflitto con tutte le prescrizioni che -dal Concilio di Toledo del 400 al Capitolare generale di Carlo Magno nel 769, al sinodo di Treviri dell'895proibivano ai chierici di portare armi o far parte dell'esercito tanto da impedire loro di diventare diaconi o vietare le preghiere in suffragio di coloro morti in guerra ${ }^{17}$. Rosino scrive le ultime volontà manu sua propria e il documento viene presentato dai parenti al Conte veneziano della città e ai giudici, entro i trenta giorni successivi alla sua morte, così come prevede lo Statuto cittadino. Ciò che sorprende è il patrimonio che mette a disposizione dei frati predicatori: un mulino, staciones poste accanto la chiesa di S. Nicola, terre, l'affitto di una casa e la riscossione dei suoi crediti, tutto in cambio di messe quotidiane e di un cero che dovrà ardere in perpetuum. Nel 1296 il chierico Barbio de Gore ${ }^{18}$, infirmus corpore, dopo aver elencato le somme destinate ai frati minori e ai frati predicatori, ai monasteri e alla Cattedrale, lascia 2 perperi per il passaggio. Anch'egli, come il Balislava, possiede una vigna e delle terre, ma dona tutto a Mirosti, figlia di una sua parente, e in particolare le concede omnia iura que michi pertinere videntur omnium ecclesiarum in quibus ius habeo ... Con cristiana carità chiude il suo testamento con una dichiarazione: qui illud rumpere voluerit, maledictionem Dei habeat et meam in eternum.

Ancora, nel 1295 Dragoe de Bocignolo ${ }^{19}$ dona al fratello scutum unum, spatam unam et baciletum unum et si frater meus iverit cum galeis habeat coraçam: il richiamo alle galee è un ulteriore riferimento al passaggio ultramarino. Al cognato lascia un arco e nell'elencare i beni posseduti riferisce di sei spade, arco e frecce, corazze. Chi non ha armi né soldi da destinare al finanziamento della guerra, partecipa comunque donando semmai p. 371 .

${ }^{15}$ Jonathan SumPTION, Monaci, santuari, pellegrini. La religione nel Medioevo, Roma, 1981,

${ }^{16}$ ASD, Testamenta, vol. 2 , ff. 7 v. -8 r.

${ }^{17}$ Sumption, Monaci, santuari, cit., p. 364 s.

${ }^{18}$ ASD, Testamenta, vol. 2 , f. 17 r.

${ }^{19} \mathrm{ASD}$, Testamenta, vol. 2, f. 14 r. 
una veges de vino meo de quinquia quinquaginta, come fa Nicola de Petniça ${ }^{20}$ nel 1297, se ci sarà pasagium generale. Nicola non lascia somme di denaro al figlio, ma gli chiede di pagare due uomini che si rechino in pellegrinaggio a S. Giacomo di Compostella.

Nell'ultimo scorcio del XIII secolo anche un artigiano raguseo, Pietro petrarius $^{21}$, devolve ben 25 perperi alla crociata. La clausola segue la decisione di affrancare la sua schiava Basilia e il piccolo schiavo (puer) Marino. Pietro ha lavorato per l'arcivescovo di Ragusa e da lui reclama un credito di 9 perperi; dichiara inoltre di dover ricevere dal ricco e potente patrizio Martolo de Tudisio, il saldo per un balcone fatto nel suo palazzo. Anche i veneziani Marco Sclapa e Marco, figlio di magister Marinus, gli sono debitori e Pietro elenca nel testamento i vari pegni conservati in casa come acconto di lavori non ancora saldati, oltre ad una notevole quantità di armi: corazze, guanti di ferro, tre spade, una balestra, arco e frecce, ben cinque scudi, panceria e finimenti per il cavallo (colaria de malo). È interessante notare che riferimenti e descrizioni di armi sono frequenti nei testamenti del XIII secolo, ma scompaiono quasi del tutto in quelli del XIV secolo.

Dei 12 testamenti del XIII secolo, ben 4 sono di donne e tutte loro destinano dai 2 ai 20 perperi alla guerra santa. Nel 1296 Slavaa $^{22}$, vedova di Marino de Binçola, madre di Agape eremita nella chiesa di Ognissanti e del prete Matteo, lega 10 perperi ad un eventuale passaggio, e se non dovesse effettuarsi i soldi dovranno essere usati per vestire i lebbrosi. Nel 1298 Stane $^{23}$, sorella di Blasio de Clima, lascia 4 perperi pro pasagium e, oltre ai diversi legati fatti ai Domenicani, chiede che venga dato un anello d'oro al braccio di S. Biagio, la reliquia del santo patrono di Ragusa; nello stesso anno, per finanziare il passaggio, Beatrice de Poveda ${ }^{24}$, moglie di Lorenzo, devolve 20 perperi specificando però che si non fuerit, pro anima, e termina con una preghiera: et rogo virum meum ut non faciat contra dictum meum testamentum ut non scandaliçetur anima mea. Ancora nel 1299 Beatrice $^{25}$, moglie di Lorenzo de Serniça, nel suo scarno testamento dà disposizione per tre perperi.

Lo stretto legame tra finanziamento per la guerra e lasciti pro anima è ancora più evidente nei documenti del XIV secolo. Qui la motivazione delle donazioni destinate al passagium viene quasi sempre espressa (pro me, pro anima mea, qui feci votum) e inoltre, al lascito per la crociata si accompagna la richiesta che vengano effettuati dei pellegrinaggi nei luoghi sacri alla cristianità.

\footnotetext{
${ }^{20} \mathrm{ASD}$, Testamenta, vol. 2, f. 26 r.

${ }^{21}$ ASD, Testamenta, vol. 2, f. 38 r.

${ }^{22}$ ASD, Testamenta, vol. 2, f. 19 r.

${ }^{23}$ ASD, Testamenta, vol. 2, f. 29 r.

${ }^{24} \mathrm{ASD}$, Testamenta, vol. 2, f. 35 r.

${ }^{25}$ ASD, Testamenta, vol. 2, f. 38 v.
} 
I 21 testamenti del XIV secolo si concentrano negli anni 1339-1348 (solo un documento è del 1362, uno è del 1363 e infine uno risale al 1384) e, anche in questo gruppo, un terzo viene fatto da nobildonne: il primo è del 1346, anno in cui Frane de Mençe ${ }^{26}$, moglie di Nicola de Georgio, stabilisce che vengano restituiti a Nicola de Mençe 75 perperi quos pro me dedit pro pasagio. Alle somme che devolve per vestire i poveri e i lebbrosi, per maritare povere gentildonne o per dotare di arredi sacri le chiese, aggiunge 50 perperi pro uno libro da dare ai frati francescani e la richiesta che due sacerdoti si rechino a S. Nicola di Bari e a S. Michele al Gargano. Due anni dopo, quando infuria l'epidemia di peste, anche Deche de Sorgo ${ }^{27}$, figlia del potente Andrea, provvede a fornire i Domenicani di paramenti e di un libro del valore di 50 perperi, somma notevole se si considera che il costo di una casa di legno si aggirava intorno ai 30 perperi.

Nel 1347 Sclava $^{28}$, moglie di Nicola de Volçio, chiede che siano dati 60 perperi alicui bone persone que vadat pro me et pro anima mea ad sanctum pasagium et si dictum pasagium esset destructum in illo anno post obitum meum, volo quod de illis denariis adiurentur maritare tres orfane punçelle de popullo pro anima mea. Nel 1348 Bune $^{29}$, figlia di Dyme de Pabora, iacens in lecto infirma, lascia 50 perperi; nello stesso anno Bilce ${ }^{30}$, moglie di Give de Gondola, ordina ...et pasagio, primo loco ubi fuerit ordinato per ecclesiam, mictatur unus homo pro anima mea quod debeat stare ibi uno anno et dentur sibi ip. LXXX. Infine, sempre nel 1348, Frane de Gondola ${ }^{31}$ lascia 100 perperi che però, se fosse desfacto, dovranno essere devoluti all'ospedale.

L'elemento comune a tutti i documenti del XIII e del XIV secolo è la certezza di un passaggio "prossimo venturo", tanto da sembrare un formulario la costante ripetizione "quando fuerit pasaçium generale", "quando erit primum pasaçium", "si pasagium fuerit" e così via, così come sembra una tariffa fissa, nel XIV secolo, la somma da devolvere che raramente è inferiore ai 100 perperi. E comune è il considerare la possibilità che venga annullato, destructum, così che quasi tutti i testatori chiedono ai loro pitropi -termine che sta ad indicare gli esecutori testamentari- di devolvere le somme assegnate ad altre cause: consacrare chiese, vestire lebbrosi, maritare fanciulle. Nicola e Martinusso de Mençe non si sottraggono a queste consuetudini.

Nicola de Mençe ${ }^{32}$, insieme al fratello Michele, aveva stretti contatti commerciali con la Bosnia, regione in cui soggiornava spesso. Nel 1348

\footnotetext{
${ }^{26}$ ASD, Testamenta, vol. 3, ff. 76 r. v.

${ }^{27}$ ASD, Doti, vol. 1 , ff. 84 r. v. -85 r.

${ }^{28}$ ASD, Testamenta, vol. 3, f. 77 r.

${ }^{29}$ ASD, Testamenta, vol. 3 , ff. 79 v. -80 r.

${ }^{30}$ ASD, Testamenta, vol. 4 , f. 25 r.

${ }^{31}$ ASD, Testamenta, vol. 5, f. 109 r.

${ }^{32}$ ASD, Doti, vol. 1, ff. 85 r. v. -86 r.
} 
chiede nel suo testamento di devolvere 100 perperi al passagium contra Saracenis infra 10 annos, e di inviare un pellegrino a Roma.

Ma è il nobile mercante Martinusso ${ }^{33}$ l'esponente più noto dei de Mençe: nel dettare nel 1347 le sue ultime volontà, destina anch'egli 100 perperi alla crociata e in più offre un cavallo e le armi a colui che sarà disposto a combattere per un anno. L'enorme patrimonio viene affidato interamente alla moglie, la potente mercantessa Filippa figlia del patrizio di Cattaro Thoma de Thoma, sic sunt allia et omnia allia sint in sua potestate, ad eccezione però di alcuni piccoli lasciti, nonché di 1000 perperi che Martinusso lega all'ospedale di Ragusa, e di ulteriori 2000 perperi che mette a disposizione di povere gentildonne e donne del popolo come dote per matrimoni. Ciò che colpisce è l'esplicita dichiarazione di gratitudine per il suocero che Martinusso fa alla moglie, quasi a giustificazione del fatto di averla nominata erede universale: cur multos denarios habui patris sui cum quibus domum meam multiplicavi et crevi.

Il testamento di Bene de Gondola, pur inserendosi nel medesimo contesto, si differenzia dagli altri per il suo accanimento nei confronti degli infedeli, siano essi turchi, saraceni o patareni di Bosnia (ad pasagium turchorum seu sarracenorum et alliud super patarenos Bosnie et quousque fuerit passagium), che apre un altro percorso di indagine: il conflitto tra Chiesa di Roma e Chiesa bosniaca.

Gondola, Binçola, Volçio, Pabora, Georgio, Menze, Babalio sono le famiglie più influenti di Ragusa, spesso unite tra loro da vincoli matrimoniali o legami d'affari. I membri di questi gruppi a partire dal XIV secolo (quando cessa il controllo veneziano sulla città nel 1358), si alternano ogni tre mesi nell'ufficio di rettore del comune: la loro è una "nobiltà mercantile" che si esplicita nel governo della città e nella gestione dei patrimoni personali ${ }^{34}$.

Ma le singole famiglie si differenziano tra loro per un aspetto in particolare: la preferenza o i legami che queste stabiliscono con i due potenti ordini monastici che si contendono la cura delle anime di Dubrovnik, Domenicani - i predicatori- e Francescani - i frati minori. Dai testamenti esaminati sembra che mentre nel XIII secolo si possa registrare un maggior peso sociale dei domenicani, nel XIV secolo sono i francescani ad attirare più lasciti per messe e "fabbriche".

La presenza dei due ordini è fondamentale per la politica papale e le mire territoriali della Serbia e dell'Ungheria sulla Bosnia.

La Chiesa era attivamente presente nella vita della comunità croata ma, come osserva il Tombor, «mentre la posizione dei sacerdoti italiani era univoca, quella del clero croato spesso era ambigua, appoggiava gli eretici, gli scismatici orientali, i catari bogomiliani, i quali, come sappiamo, erano

\footnotetext{
${ }^{33}$ ASD, Testamenta, vol. 3, ff. 75 v. - $76 \mathrm{r}$

${ }^{34}$ Per un panorama sulle istituzioni dalmate e su Ragusa cfr. Tibor TOMBOR, La formazione e lo sviluppo dell'autonomia comunale delle città dalmate nel Medioevo, in Atti e Memorie della Società Dalmata di Storia Patria, XIII, Venezia, 1985, pp. 241-274.
} 
serbi, bulgari, bosniaci. Si manifestava una specie di non ancora chiaramente concepito panslavismo $»^{35}$.

La lotta al dualismo, o manicheismo, fu particolarmente accesa nel XIII secolo e la Chiesa di Roma invitava a prendere la Crux cismarina «poichè gli eretici hanno abbandonato la vera fede con cognizione di causa e rappresentano con il loro proselitismo un immenso pericolo per le anime ${ }^{36}$. L'Evangelisti sottolinea che «eretici ed infedeli, anche a seguito dello sforzo canonistico e della testualità conciliare espressa tra i cinque concili lateranensi (1179-1517) passando per i concili lionesi e di Vienne (1245-1312), non sono solo coloro che che rifiutano su un piano teologico l'obbedienza ai dogmi della fede della cristianità romana $\mathrm{o}$ i musulmani d'Oltremare: sono molti di più $\mathrm{e}$ assai diversificati nella loro fisionomia. Sono concretamente e specificatamente gli ebrei all'interno delle comunità politiche ed istituzionali che stanno crescendo, anche in termini identitari, all'interno della res publica christiana; sono i soggetti che esprimono forme di dissenso pauperistico e radicale che a volte si salda con autorità civili in competizione territoriale e politica con altri domini; sono tutti coloro che utilizzano pratiche creditizie non controllate o autorizzate dai consacrati e dalla Chiesa di Roma.... ${ }^{37}$.

Il problema è che non si può affermare con certezza fino a che punto per la Bosnia si possa parlare realmente di eresia, giacché nessun papa indica quali sono gli errori dottrinali in cui incorrono i fedeli, ma si puô ipotizzare che se la fedeltà a Roma faceva di un uomo un cattolico, la fedeltà alla gerarchia della chiesa di Bosnia lo rendeva un cristiano bosniaco ${ }^{38}$.

Gli studi più recenti hanno evidenziato che in realtà, a dispetto dell'allarmismo che traspare dalle lettere dei papi e nei documenti dell'Inquisizione circa il diffondersi dell'eresia in Bosnia, alla popolazione e ai ministri del culto potevano imputarsi solo grossolani errori dottrinali, probabilmente dovuti al fatto che la chiesa bosniaca officiava in lingua croata, e, inoltre, pur riconoscendo l'autorità della Santa Sede, aveva mantenuto il diritto di eleggere i propri vescovi ${ }^{39}$.

Si è acutamente evidenziato come molti degli studiosi che si sono occupati dell'eresia bosniaca abbiano sottovalutato il contesto politico e sociale: tutta la storia religiosa di questa regione ha luogo in ambito rurale e basterebbe, solo questo, a differenziarla dai movimenti ereticali del nord Italia e della Francia del sud, tipicamente urbani. Le città che si svilupparono in Bosnia solo a partire dalla seconda metà del XIV secolo, divennero i centri di una restaurazione del cattolicesimo, soprattutto a causa della presenza dei

\footnotetext{
${ }^{35}$ TOMBor, La formazione e lo sviluppo, cit., p. 255.

${ }^{36}$ CONTAMINE, cit., p.378.

${ }^{37}$ Paolo Evangelisti, Martirio volontario e ideologia della Crociata. Formazione e irradiazione dei modelli francescani a partire dalle matrici altomedievali, in «Cristianesimo nella Storia», 27, Bologna, 2006, pp. 161-248, p. 201.

${ }^{38} \mathrm{John}$ V.A. FInE, The Bosnian Church: a new interpretation, Boulder, New York-London, 1975, p. IV.

${ }^{39}$ Stanko GuldeSCU, History of Medieval Croatia, The Hague, 1964, p. 247 s.
} 
cattolici mercanti stranieri (soprattutto di Dubrovnik), che fondarono lì importanti colonie - vitali per i loro commerci-chiese, ma che soprattutto attirarono i Francescani a cui offrirono sostegno e protezione.

Come ha sottolineato il Fine «spesso si è affermata una sorta di regola generale delle società medievali e cioè che la fede religiosa dei governanti definisca anche quella dei sudditi» ${ }^{40}$. Nel nostro caso, i principi della Bosnia medievale furono tolleranti, se non indifferenti, alle questioni religiose. All'interno dei Balcani prevalse infatti l'ideale della piena armonia tra potere spirituale e potere temporale, ognuno dei quali svolgeva i compiti assegnatigli nella propria $\operatorname{sfera}^{41}$.

La religione divenne un fattore decisivo quando i potentati stranieri, in particolare i re ungheresi - re di Croazia e Dalmazia - e serbi, decisero di assoggettare la Bosnia, abitata dai «dannati bogomili» ed «eretici tre volte maledetti»; solo a metà XIV secolo il clima prodotto dalla minaccia turca permise di ricompattare le fila dei "cristiani" 42 .

Tra XIII e XIV secolo le pretese dinastiche nell'area balcanica, i contrasti e gli interessi sulla costa adriatica di Genova e Venezia, le alleanze tra Venezia e Serbia e tra Genova e Ungheria ${ }^{43}$, vengono abilmente celate dietro denunce di sospette eresie e guerre per la difesa della fede. Ricordiamo, solo per fare qualche esempio, l'informativa ricevuta dal papa Innocenzo III nel 1199 sulla presunta diffusione di eresie nei territori ungheresi di Bosnia; o la richiesta fatta nel 1200 dallo stesso pontefice al re di Ungheria, di cacciare i patareni di Spalato e Traù rifugiatisi in Bosnia, invito che costrinse il ban bosniaco a organizzare in fretta una delegazione che rassicurasse il papa sulla sua fedeltà.

Già alla fine del XII secolo la Serbia si era avvicinata alla Chiesa di Roma, cosicché la conversione del loro re Stefano aveva suggerito a papa Innocenzo III di proclamare la prima crociata contro gli eretici bogomiliani di Bosnia: ma i re serbi erano troppo vicini alla Chiesa ortodossa e la Santa Sede iniziò a fare affidamento sull'aiuto dei cattolici re croato-ungheresi4 ${ }^{44}$.

E' del 1234 un nuovo tentativo di indire una crociata in vista di una riorganizzazione della chiesa cattolica di Bosnia. Nelle lettere che Gregorio IX invia a Coloman, duca di Croazia, c'è la promessa di indulgenze per coloro che combatteranno gli eretici e così dal 1235 al 1238 si lottò per la "vera fede". Gregorio considerò la guerra un successo, ma a ben guardare fu anch'essa una guerra di conquista fatta esplodere e portata a termine dall'Ungheria, con il sostegno dei domenicani.

\footnotetext{
${ }^{40}$ FInE, The Bosnian Church, cit., p. 29

${ }^{41}$ Sima Circović, I Serbi nel Medioevo, Milano, 1992, p. 58.

${ }^{42}$ Circović, I Serbi, cit., p. 61.

${ }^{43} \mathrm{Cfr}$. a riguardo il lavoro di Tibor TOMBOR, L'alleanza della repubblica di Genova con l'Ungheria nel secolo XIV contro la repubblica di Venezia per il dominio della Dalmazia e l'impero del mare, "Atti e Memorie della Società Dalmata di Storia Patria», XI (Venezia, 1983), pp. 221-261.

${ }^{44}$ GulDESCU, History of Medieval Croatia, cit., p. 250
} 
Questa serie di campagne militari, definite dal Flori "crociate degenerate" e tutte volte a debellare le più diverse eresie, da quella albigese a quella hussita, consentono ai cavalieri di restare in Occidente a combattere guerre "di carattere locale" 45

Tra il 1246 e il 1247 Innocenzo IV cercò di organizzare una nuova spedizione, ma vi rinunciò nel 1248 preferendo inviare presso il ban Ninoslav un frate francescano che potesse fornirgli una opinione neutrale sull'ortodossia del governante e, soprattutto, non influenzata dall'Ungheria, fortemente sostenuta dall'ordine domenicano. Fu una netta presa di posizione contro gli intrighi e l'eccessiva ingerenza dei frati predicatori. Innocenzo - dopo aver ricevuto da Ninoslav rassicurazioni circa la sua fedeltà alla Chiesa di Romachiese all'Ungheria di cessare tutte le ostilità contro la Bosnia.

Gli allarmi contro il diffondersi delle eresie lanciati dai papi nel 1280, nel 1298 e nel 1303, spiegano lo zelo dei Francescani e dei Domenicani nel pubblicizzare le crociate e ci permettono di spiegare la concentrazione di lasciti per il passagium che abbiamo registrato nell'ultimo decennio del XIII secolo.

Riferimenti alla lotta contro i movimenti ereticali si trovano ancora nei documenti pontifici del primo trentennio del 1300.

Ma nel 1337 cambia radicalmente l'atteggiamento della Santa Sede: papa Benedetto XII obbliga i principi e nobili di Krbava, Senj, Bribir, Knin e Croazia a dare supporto armato ai Francescani, ormai non più in grado di proseguire nel loro compito di inquisitori in Bosnia ${ }^{46}$. In seguito la strategia papale fu quella di utilizzare la diplomazia e la missione francescana: sembra che il generale dell'Ordine, Gerardo Odini, riuscisse a convertire al cattolicesimo l'ortodosso Stjepan Kotromanic tra il 1340 e il 1342, ma alcune fonti ragusee attribuiscono tale conversione al canonico di Dubrovnik Domagna de Volzio de Babalio. A lui il nostro testatore Bene de Gondola, così ansioso di sovvenzionare qualsiasi passaggio, quousque fuerit, aveva lasciato 5 perperi ed è lui che la nobile Bune de Pabora aveva scelto come esecutore delle sue ultime volontà. E' certo comunque che Domagna de Babalio aiutò i Francescani nella loro missione di fondare il vicariato di Bosnia.

Nessuna fonte fa più menzione di chiamate alle armi fino al 1357. I testamenti ragusei che ritroviamo per gli anni 1346-1348, ad eccezione di due atti in cui è evidente che i soldi erano già stati offerti per la crociata del 1339 (Frane de Georgio e Draseno Nicucich mercante), riflettono dunque solo il timore o la speranza di un nuovo "passaggio". Infatti, non bisogna dimenticare, tra l'altro, che tra la fine del XIII secolo e durante quello successivo, la città fu tra i principali mercati di schiavi e che potevano essere ridotti in

\footnotetext{
${ }^{45}$ Jean FLORI, Le crociate, Bologna, 2003, p. 121 ss.

${ }^{46}$ Evangelisti, Martirio volontario, cit., p. 232: «... l'azione francescana si esplica nei confronti di ogni forma di infidelitas. I francescani combattono e muoiono da Vilnius a Tolosạ, dal Marocco, alla Georgia. Il paganesimo lituano o prussiano, l'eresia catara, l'ortodossia georgiana e l'islam sono obiettivi parimenti rilevanti. In siffatta prospettiva, evidentemente, nelle cronạche ufficiali dell'Ordine sono martiri francescani a tutti gli effetti non solo i Minori predicatori, ma anche i Minori inquisitori».
} 
schiavitù solo i non battezzati o gli eretici, in particolare i patarini di Bosnia, i quali venivano venduti soprattutto ai molti catalani presenti a Dubrovnik fin dal $1281^{47}$.

Le forti tensioni per la minaccia turca, i tentativi di ricondurre Chiesa di Roma e Chiesa ortodossa ad unitatem Sancte Romane Ecclesie, la lotta alle eresie, erano tutti buoni motivi per indire crociate che spesso rimasero solo tentativi. Insieme ai Francescani, furono inviati dai papi anche Domenicani, Agostiniani e Carmelitani, tutti a predicare la giusta guerra.

Nella seconda metà del XIV secolo l'attenzione di Roma si rivolse soprattutto ai Turchi. Da questo momento in avanti "lo scopo dell'ancor viva ma già logorata idea di crociata non era più la liberazione del Santo Sepolcro e della Terra Santa dai pagani, ma la cacciata dei Turchi dalla penisola balcanica nell'Asia Minore, cioè la difesa dei cristiani balcanici" ${ }^{48}$.

Gli ultimi testamenti che registrano donazioni per la crociata risalgono agli anni 60 del Trecento. Nel 1362 Vali de Goçe ${ }^{49}$ lascia 100 perperi alicui bone persone qui vadat ad pasagium pro anima mea quia feci votum e, pro anima, chiede pellegrinaggi a Roma, Bari e Montesantangelo. Insolitamente dona alla moglie, tra le altre cose, spade, scudi, la corazza e i coltelli de feriendo. L'anno successivo, nel 1363, Marino chapellar $^{50}$ chiede che venga venduta la sua vigna e che col ricavato venga mandato un uomo al passaggio e ad Assisi. La colpa di aver "maltolletto" viene risarcita con 200 perperi e con numerose donazioni alle fratiglie di cui è socio.

In conclusione è più che condivisibile l'opinione che a partire dal XIII secolo sia paradossale parlare di una conflittualità tra spiritualia e militaria. L'eterna lotta tra coorti celesti e legioni diaboliche da metaforica diventò reale e "portò in certo qual modo alla sacralizzazione della guerra, a un rafforzamento del prestigio dei guerrieri e del mestiere delle armi. La battaglia fu sentita come un'attività pienamente degna di lode e propedeutica alla salvezza, il "valore" militare contro coloro fuori dalla Chiesa, come una virtù più che umana" ${ }^{51}$. Lo spazio medievale era nettamente diviso tra cristiani e non cristiani e questo superava il concetto di popoli e nazioni: aderire alla missione di Roma tesa a convertire, anche con la forza, tutti coloro che si trovavano al di fuori della communitas christianorum ribadiva l'appartenenza a pieno titolo a quello spazio.

\footnotetext{
${ }^{47} \mathrm{Cfr}$. i lavori di Desanka KovaČEviĆ-KoJIĆ, Il commercio raguseo di terraferma nel Medioevo, in DI VITTORIO, cit., pp. 61-78; Nenad FEJIC, Ragusei e Spagnoli nel Medioevo. Luci ed ombre di un rapporto commerciale, in DI VITTORIO, cit., pp. 79-100. Fejic calcola in 323 unità i catalani che entrarono a Ragusa dal' 1281 al 1499.

${ }^{48}$ Vassil GuUZeleV, La Chiesa cattolica di Roma e la Bulgaria nel periodo della conquista turca della penisola balcanica, in Laura BALLETTO, Oriente e Occidente tra Medioevo ed Età Moderna. Studi in onore di Geo Pistarino, Genova, 1997, p. 417. Cfr. anche Alain DUCELLIER, Cristiani d'Oriente e Islam nel Medioevo, Torino, 2001, in particolare cap. 12.

${ }^{49}$ ASD, Testamenta, vol. 4 , ff. 56 v. 57 r.

${ }^{50} \mathrm{ASD}$, Testamenta, vol. 5, ff. 245 r. v.

${ }^{51}$ Contamine, La guerra, cit., pp. 407 ss.
} 
Il cristianesimo e la guerra, la Chiesa e i militari, non sono più concetti antitetici, ma simbiotici e necessari l'uno all'altro ${ }^{52}$. I testamenti analizzati avvalorano quanto detto ma sono, soprattutto, la testimonianza di una società in cui né la cavalleria, né il clero, né la borghesia sembrano più desiderose di sacrifici: piuttosto sono desiderose di una salvezza economica anziché di quella spirituale ${ }^{53}$.

Data di ricezione dell'articolo: maggio 2008.

Data di accettazione e versione finale: giugno 2008.

${ }^{52}$ Ibidem, p. 399.

${ }^{53}$ GuUZELEV, La Chiesa cattolica di Roma, cit., p. 417. 
I MERCANTI RAGUSEI E LE CROCIATE DEL TARDO MEDIOEVO

TABELLA RIASSUNTIVA

TESTAMENTI RELATIVI AL PASSAGIUM

\begin{tabular}{|c|c|c|c|c|c|c|c|}
\hline ANNO & NOME & $\begin{array}{l}\text { FAMI- } \\
\text { GLIA }\end{array}$ & $\begin{array}{l}\text { CONDI- } \\
\text { ZIONI }\end{array}$ & $\begin{array}{l}\text { ME- } \\
\text { STIERE }\end{array}$ & $\begin{array}{l}\text { PELLE- } \\
\text { GRINI }\end{array}$ & $\begin{array}{l}\text { MOTI- } \\
\text { VAZIO- } \\
\text { NE }\end{array}$ & $\begin{array}{l}\text { LASCI- } \\
\text { TO }\end{array}$ \\
\hline 1284 & Elias & de Rasti & Infermo & Mercante & & & $\begin{array}{l}\text { perperi } \\
20 \text { e pan- } \\
\text { ceria }\end{array}$ \\
\hline 1295 & Rosino & Balislava & & Chierico & $\begin{array}{l}1 \text { uomo } \\
\text { armato }\end{array}$ & & $\begin{array}{l}\text { Pance- } \\
\text { ria, spa- } \\
\text { ta, capi- } \\
\text { lina, scu- } \\
\text { tum }\end{array}$ \\
\hline 1295 & Dragoe & $\begin{array}{l}\text { de Bo- } \\
\text { cignolo }\end{array}$ & Infermo & & $\begin{array}{l}\text { Fratello, } \\
\text { si iverit } \\
\text { cum ga- } \\
\text { leis }\end{array}$ & & $\begin{array}{l}\text { Scudo, } \\
\text { spada, } \\
\text { bacile- } \\
\text { tum }\end{array}$ \\
\hline 1296 & Slava & $\begin{array}{l}\text { de Bin- } \\
\text { çola }\end{array}$ & & & & & $\begin{array}{l}\text { perperi } \\
10\end{array}$ \\
\hline 1296 & Barbio & de Gore & Infermo & Chierico & & & perperi 2 \\
\hline 1297 & Ursacius & $\begin{array}{l}\text { de Gule- } \\
\text { rico }\end{array}$ & & & & & $\begin{array}{l}\text { perperi } \\
14 \text {, armi; } \\
\text { pp. } 8 \text { per } \\
\text { il padre }\end{array}$ \\
\hline 1297 & Nicola & $\begin{array}{l}\text { de Petni- } \\
\text { ça }\end{array}$ & Infermo & & 1 uomo & & $\begin{array}{l}\text { Una ve- } \\
\text { ges de } \\
\text { vino }\end{array}$ \\
\hline 1298 & Luboe & de Mica & Infermo & & & & $\begin{array}{l}\text { perperi } \\
10\end{array}$ \\
\hline 1298 & Beatrice & $\begin{array}{l}\text { de Pove- } \\
\text { da }\end{array}$ & & & & & $\begin{array}{l}\text { perperi } \\
20\end{array}$ \\
\hline 1298 & Stana & de Clime & & & & & perperi 4 \\
\hline 1299 & Pietro & & & Lapicida & & & $\begin{array}{l}\text { perperi } \\
25\end{array}$ \\
\hline 1299 & Beatrice & $\begin{array}{l}\text { de Serni- } \\
\text { ça }\end{array}$ & & & & & perperi 3 \\
\hline 1339 & $\begin{array}{l}\text { Don Cle- } \\
\text { mente }\end{array}$ & $\begin{array}{l}\text { de Gan- } \\
\text { gullis }\end{array}$ & & Prete & & & $\begin{array}{l}\text { perperi } \\
20 \text { quan- } \\
\text { do fuerit }\end{array}$ \\
\hline 1340 & Junio & $\begin{array}{l}\text { de Vol- } \\
\text { cassio }\end{array}$ & & $\begin{array}{l}\text { Mercan- } \\
\text { te, am- } \\
\text { basciato- } \\
\text { re }\end{array}$ & & & $\begin{array}{l}\text { perperi } \\
25 ; \text { pp. } \\
70 \text { per il } \\
\text { padre }\end{array}$ \\
\hline
\end{tabular}




\begin{tabular}{|c|c|c|c|c|c|c|c|}
\hline 1346 & Franca & $\begin{array}{l}\text { de Men- } \\
\text { çe }\end{array}$ & Inferma & & & & $\begin{array}{l}\text { perperi } \\
65\end{array}$ \\
\hline 1347 & Marcolo & $\begin{array}{l}\text { de Zrie- } \\
\text { va }\end{array}$ & & Mercante & 1 uomo & $\begin{array}{l}\text { Pro ani- } \\
\text { ma mea }\end{array}$ & $\begin{array}{l}\text { perperi } \\
100\end{array}$ \\
\hline 1347 & Draseno & Nicuçich & Infermo & Mercante & 2 uomini & Pro me & $\begin{array}{l}\text { perperi } \\
300\end{array}$ \\
\hline 1347 & $\begin{array}{l}\text { Marti- } \\
\text { nusso }\end{array}$ & $\begin{array}{l}\text { de Men- } \\
\text { çe }\end{array}$ & Infermo & Mercante & & & $\begin{array}{l}\text { perperi } \\
100 \text { e } \\
\text { cavallo e } \\
\text { armi, per } \\
\text { un anno. }\end{array}$ \\
\hline 1347 & Sclava & $\begin{array}{l}\text { de Vol- } \\
\text { zio }\end{array}$ & Inferma & & $\begin{array}{l}\text { Bona } \\
\text { persona }\end{array}$ & Pro me & $\begin{array}{l}\text { perperi } \\
60\end{array}$ \\
\hline 1348 & Deche & de Sorgo & Sana & & $\begin{array}{l}\text { Una bo- } \\
\text { na perso- } \\
\text { na }\end{array}$ & $\begin{array}{l}\text { Pro ani- } \\
\text { ma mea }\end{array}$ & $\begin{array}{l}\text { Et ibi } \\
\text { stet per } \\
\text { unum } \\
\text { annum } \\
\text { tantum } \\
\text { quan- } \\
\text { tum.. }\end{array}$ \\
\hline 1348 & Gabriel & de Gleda & & & & & $\begin{array}{l}\text { Per una } \\
\text { femina } \\
\text { che mi le } \\
\text { de per } \\
\text { mandare }\end{array}$ \\
\hline 1348 & Vole & $\begin{array}{l}\text { de Vol- } \\
\text { zio }\end{array}$ & Sano & Mercante & & $\begin{array}{l}\text { Pro ani- } \\
\text { ma mea }\end{array}$ & $\begin{array}{l}\text { perperi } \\
42\end{array}$ \\
\hline 1348 & Mare & de Dabro & & & 1 uomo & & $\begin{array}{l}\text { perperi } \\
100\end{array}$ \\
\hline 1348 & Lauriça & de Sorgo & & Mercante & 1 uomo & & $\begin{array}{l}\text { perperi } \\
100\end{array}$ \\
\hline 1348 & Frave & $\begin{array}{l}\text { de Gon- } \\
\text { dola }\end{array}$ & Infermo & & & Per me & $\begin{array}{l}\text { perperi } \\
100 \text { e se } \\
\text { fosse } \\
\text { desfacto } \\
\text { alo spi- } \\
\text { dal }\end{array}$ \\
\hline 1348 & Nicholla & $\begin{array}{l}\text { de Men- } \\
\text { çe }\end{array}$ & & & & & $\begin{array}{l}\text { perperi } \\
100 \text { con- } \\
\text { tro Sara- } \\
\text { cenis } \\
\text { infra } 10\end{array}$ \\
\hline 1348 & Bene & $\begin{array}{l}\text { de Gon- } \\
\text { dola }\end{array}$ & & & & & $\begin{array}{l}\text { perperi } \\
140\end{array}$ \\
\hline 1348 & Sime & de Grede & & 1 uomo & & & $\begin{array}{l}\text { perperi } \\
25\end{array}$ \\
\hline
\end{tabular}




\begin{tabular}{|l|l|l|l|l|l|l|l|}
\hline 1348 & Bune & $\begin{array}{l}\text { de Pabo- } \\
\text { ra }\end{array}$ & Infermo & & 1 uomo & $\begin{array}{l}\text { Pro ani- } \\
\text { ma mea }\end{array}$ & $\begin{array}{l}\text { perperi } \\
50\end{array}$ \\
\hline 1348 & Bilce & $\begin{array}{l}\text { de Gon- } \\
\text { dola }\end{array}$ & Sana & & 1 uomo & $\begin{array}{l}\text { Pro ani- } \\
\text { ma mea }\end{array}$ & $\begin{array}{l}\text { perperi } \\
80, \text { pri- } \\
\text { mo loco } \\
\text { ubi fuerit } \\
\text { ordina- } \\
\text { tum }\end{array}$ \\
\hline 1362 & Vali & de Goçe & & & & $\begin{array}{l}\text { Quia feci } \\
\text { votum }\end{array}$ & $\begin{array}{l}\text { perperi } \\
100\end{array}$ \\
\hline 1363 & Marino & & & $\begin{array}{l}\text { Cha- } \\
\text { pellar }\end{array}$ & 1 uomo & & $\begin{array}{l}\text { Vendita } \\
\text { della } \\
\text { vigna }\end{array}$ \\
\hline
\end{tabular}

